\title{
Maximum Entropy Models for FrameNet Classification
}

\author{
Michael Fleischman, Namhee Kwon and Eduard Hovy \\ USC Information Sciences Institute \\ 4676 Admiralty Way \\ Marina del Rey, CA 90292-6695 \\ \{fleisch, nkwon, hovy \}@ISI.edu
}

\begin{abstract}
The development of FrameNet, a large database of semantically annotated sentences, has primed research into statistical methods for semantic tagging. We advance previous work by adopting a Maximum Entropy approach and by using previous tag information to find the highest probability tag sequence for a given sentence. Further we examine the use of sentence level syntactic pattern features to increase performance. We analyze our strategy on both human annotated and automatically identified frame elements, and compare performance to previous work on identical test data. Experiments indicate a statistically significant improvement $(\mathrm{p}<0.01)$ of over $6 \%$.
\end{abstract}

\section{Introduction}

Recent work in the development of FrameNet, a large database of semantically annotated sentences, has laid the foundation for statistical approaches to the task of automatic semantic classification.

The FrameNet project seeks to annotate a large subset of the British National Corpus with semantic information. Annotations are based on Frame Semantics (Fillmore, 1976), in which frames are defined as schematic representations of situations involving various frame elements such as participants, props, and other conceptual roles.

In each FrameNet sentence, a single target predicate is identified and all of its relevant frame elements are tagged with their semantic role (e.g., Agent, Judge), their syntactic phrase type (e.g.,
NP, PP), and their grammatical function (e.g., external argument, object argument). Figure 1 shows an example of an annotated sentence and its appropriate semantic frame.

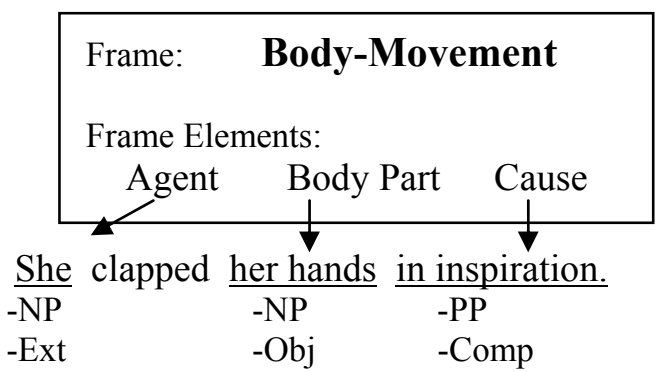

Figure 1. Frame for lemma "clap" shown with three core frame elements and a sentence annotated with element type, phrase type, and grammatical function.

As of its first release in June 2002, FrameNet has made available 49,000 annotated sentences. The release contains 99,000 annotated frame elements for 1462 distinct lexical predicates (927 verbs, 339 nouns, and 175 adjectives).

While considerable in scale, the FrameNet database does not yet approach the magnitude of resources available for other NLP tasks. Each target predicate, for example, has on average only 30 sentences tagged. This data sparsity makes the task of learning a semantic classifier formidable, and increases the importance of the modeling framework that is employed.

\section{Related Work}

To our knowledge, Gildea and Jurafsky (2002) is the only work to use FrameNet to build a statistically based semantic classifier. They split the problem into two distinct sub-tasks: frame element identification and frame element classification. In the identification phase, syntactic information is extracted from a parse tree to learn the boundaries 
of the frame elements in a sentence. In the classification phase, similar syntactic information is used to classify those elements into their semantic roles.

In both phases Gildea and Jurafsky (2002) build a model of the conditional probabilities of the classification given a vector of syntactic features. The full conditional probability is decomposed into simpler conditional probabilities that are then interpolated to make the classification. Their best performance on held out test data is achieved using a linear interpolation model:

$$
p(r \mid x)=\sum_{i=0}^{m} \alpha_{i} p\left(r \mid x_{i}\right)
$$

where $r$ is the class to be predicted, $x$ is the vector of syntactic features, $x_{i}$ is a subset of those features, $\alpha_{i}$ is the weight given to that subset conditional probability (as determined using the EM algorithm), and $m$ is the total number of subsets used. Using this method, they report a test set accuracy of $78.5 \%$ on classifying semantic roles and precision/recall scores of $.726 / .631$ on frame element identification.

We extend Gildea and Jurafsky (2002)'s initial effort in three ways. First, we adopt a maximum entropy (ME) framework in order to learn a more accurate classification model. Second, we include features that look at previous tags and use previous tag information to find the highest probability semantic role sequence for a given sentence. Finally, we examine sentence-level patterns that exploit more global information in order to classify frame elements. We compare the results of our classifier to that of Gildea and Jurafsky (2002) on matched test sets of both human annotated and automatically identified frame elements.

\section{Semantic Role Classification}

Training (36,993 sentences / 75,548 frame elements), development (4,000 sentences / 8,167 frame elements), and held out test sets (3,865 sentences / 7,899 frame elements) were obtained in order to exactly match those used in Gildea and Jurafsky (2002) ${ }^{1}$. In the experiments presented below, features are extracted for each frame element in a sentence and used to classify that ele-

\footnotetext{
${ }^{1}$ Data sets (including parse trees) were obtained from Dan
} Gildea via personal communication. ment into one of 120 semantic role categories. The boundaries of each frame element are given based on the human annotations in FrameNet. In Section 4, experiments are performed using automatically identified frame elements.

\subsection{Features}

For each frame element, features are extracted from the surface text of the sentence and from an automatically generated syntactic parse tree (Collins, 1997). The features used are described below:

- Target predicate (tar): Although there may be many predicates in a sentence with associated frame elements, classification operates on only one target predicate at a time. The target predicate is the only feature that is not extracted from the sentence itself and must be given by the user. Note that the frame which the target predicate instantiates is not given, leaving any word sense ambiguities to be handled implicitly by the classifier. ${ }^{2}$

- Phrase type (pt): The syntactic phrase type of the frame element (e.g. NP, PP) is extracted from the parse tree of the sentence by finding the constituent in the tree whose boundaries match the human annotated boundaries of the element. In cases where there exists no constituent that perfectly matches the element, the constituent is chosen which matches the largest text span of the element and has the same leftmost boundary.

- Syntactic head (head): The syntactic heads of the frame elements are extracted from the frame element's matching constituent (as described above) using a heuristic method described by Michael Collins. ${ }^{3}$ This method extracts the syntactic heads of constituents; thus, for example, the second frame element in Figure 1 has head "hands," while the third frame element has head "in."

- Logical Function (If): A simplification of the grammatical function annotation (see section 1 ) is extracted from the parse tree. Unlike the

\footnotetext{
${ }^{2}$ Because of the interaction of head word features with the target predicate, we suspect that ambiguous lexical items do not account for much error. This question, however, will be addressed explicitly in future work.

${ }^{3} \mathrm{http}: / /$ www.ai.mit.edu/people/mcollins/papers/heads
} 
Table 1. Feature sets used in ME frame element classifier. Shows individual feature sets, example feature function from that set, and total number of feature functions in the set. Examples taken from frame element "in inspiration," shown in Figure 1.

\begin{tabular}{|c|c|c|c|}
\hline Number & Feature Set & Example function & $\begin{array}{c}\text { Number of Functions } \\
\text { in Feature Set }\end{array}$ \\
\hline 0 & $f(r$, tar $)$ & $f($ CAUSE, "clap" $)=1$ & 6,518 \\
\hline 1 & $f(r, t a r, p t)$ & $f($ CAUSE, "clap", $P P)=1$ & 12,030 \\
\hline 2 & $f(r, t a r, p t, l f)$ & $f($ CAUSE, "clap", PP, other $)=1$ & 14,615 \\
\hline 3 & $f(r, p t, p o s$, voice $)$ & $f($ CAUSE, NP, "clap", active $)=1$ & 1,215 \\
\hline 4 & $f(r, p t$, pos, voice, tar $)$ & $f($ CAUSE, $P P$, after, active, "clap" $)=1$ & 15,602 \\
\hline 5 & $f(r, h e a d)$ & $f($ CAUSE, "in") $=1$ & 18,504 \\
\hline 6 & $f(r$, head, tar $)$ & $f($ CAUSE, "in", "clap") $=1$ & 38,223 \\
\hline 7 & $f(r$, head, tar, $p t)$ & $f($ CAUSE, "in", "clap", $P P)=1$ & 39,740 \\
\hline 8 & $f(r$, order, syn $)$ & $\begin{array}{l}f(\text { CAUSE, } 2, \\
[N P \text {-Ext }, \text { Target }, N P-O b j, P P \text {-other }])=1\end{array}$ & 13,228 \\
\hline 9 & $f(r$, tar, order, syn $)$ & $\begin{array}{l}f(\text { CAUSE, "clap", } 2, \\
[N P \text {-Ext }, \text { Target }, N P-O b j, P P \text {-other }])=1\end{array}$ & 40,580 \\
\hline 10 & $f\left(r, r_{-}-1\right)$ & $f(C A U S E, B O D Y P A R T)=1$ & 1,158 \\
\hline 11 & $f\left(r, r_{-}-1, r_{-}-2\right)$ & $f($ CAUSE, BODYPART, AGENT) $=1$ & 2,030 \\
\hline \multicolumn{3}{|c|}{ Total Number of Features: } & 203,443 \\
\hline
\end{tabular}

full grammatical function, the if can have only one of three values: external argument, object argument, other. A node is considered an external argument if it is an ancestor of an $\mathrm{S}$ node, an object argument if it is an ancestor of a VP node, and other for all other cases. This feature is only applied to frame elements whose phrase type is NP.

- Position (pos): The position of the frame element relative to the target (before, after) is extracted based on the surface text of the sentence.

- Voice (voice): The voice of the sentence (active, passive) is determined using a simple regular expression passed over the surface text of the sentence.

- Order (order): The position of the frame element relative to the other frame elements in the sentence. For example, in the sentence from Figure 1, the element "She" has order $=0$, while "in inspiration" has order=2.

- Syntactic pattern (pat): The sentence level syntactic pattern of the sentence is generated by looking at the phrase types and logical functions of each frame element in the sentence. For example, in the sentence: "Alexandra bent her head;" "Alexandra" is an external argument Noun Phrase, "bent" is a target predicate, and "her head" is an object argument Noun Phrase. Thus, the syntactic pattern associated with the sentence is [NP-ext, target, NP-obj].
These syntactic patterns can be highly informative for classification. For example, in the training data, a syntactic pattern of [NPext, target, NP-obj] given the predicate bend was associated $100 \%$ of the time with the Frame Element pattern: "AGENT TARGET BODYPART."

- Previous role $\left(\mathbf{r}_{-} \boldsymbol{n}\right)$ : Frame elements do not occur in isolation, but rather, depend very much on the other elements in a sentence. This dependency can be exploited in classification by using the semantic roles of previously classified frame elements as features in the classification of a current element. This strategy takes advantage of the fact that, for example, if a frame element is tagged as an AGENT it is highly unlikely that the next element will also be an AGENT.

The previous role feature indicates the classification that the $n$-previous frame element received. During training, this information is provided by simply looking at the true classes of the frame element occurring $n$ positions before the target element. During testing, hypothesized classes of the $n$ elements are used and Viterbi search is performed to find the most probable tag sequence for a sentence.

\subsection{Maximum Entropy}

ME models implement the intuition that the best model will be the one that is consistent with the set of constrains imposed by the evidence, but other- 
wise is as uniform as possible (Berger et al., 1996). We model the probability of a semantic role $r$ given a vector of features $x$ according to the ME formulation below:

$$
p(r \mid x)=1 / Z_{x} \exp \left[\sum_{i=0}^{n} \lambda_{\mathrm{i}} f_{i}(r, x)\right]
$$

Here $Z_{\mathrm{x}}$ is a normalization constant, $f_{i}(r, x)$ is a feature function which maps each role and vector element (or combination of elements) to a binary value, $n$ is the total number of feature functions, and $\lambda_{i}$ is the weight for a given feature function. The final classification is just the role with highest probability given its feature vector and the model.

The feature functions that we employ can be divided into feature sets based upon the types and combinations of features on which they operate. Table 1 lists the feature sets that we use, as well as the number of individual feature functions they contain. The feature combinations were chosen based both on previous work and trial and error. In future work we will examine more principled feature selection techniques.

It is important to note that the feature functions described here are not equivalent to the subset conditional distributions that are used in the Gildea and Jurafsky model. ME models are log-linear models in which feature functions map specific instances of syntactic features and classes to binary values (e.g., if a training element has head="in" and role $=$ CAUSE, then, for that element, the feature function $f$ (CAUSE, "in") will equal 1). Thus, ME is not here being used as another way to find weights for an interpolated model. Rather, the ME approach provides an overarching framework in which the full distribution of semantic roles given syntactic features can be modeled.

We train the ME models using the GIS algorithm (Darroch and Ratcliff, 1972) as implemented in the YASMET ME package (Och, 2002). We use the YASMET MEtagger (Bender et al., 2003) to perform the Viterbi search. The classifier was trained until performance on the development set ceased to improve. Feature weights were smoothed using Gaussian priors with mean 0 (Chen and Rosenfeld, 1999). The standard deviation of this distribution was optimized on the development set for each experiment.

\subsection{Experiments}

We present three experiments in which different feature sets are used to train the ME classifier. The first experiment uses only those feature combinations described in Gildea and Jurafsky (2002) (feature sets 0-7 from Table 1). The second experiment uses a super set of the first and incorporates the syntactic pattern features described above (feature sets 0-9). The final experiment uses the previous tags and implements Viterbi search to find the best tag sequence (feature sets 0-11).

We further investigate the effect of varying two aspects of classifier training: the standard deviation of the Gaussian priors used for smoothing, and the number of sentences used for training. To examine the effect of optimizing the standard deviation, a range of values was chosen and a classifier was trained using each value until performance on a development set ceased to improve.

To examine the effect of training set size on performance, five data sets were generated from the original set with $36,367,3674,7349$, and 24496 sentences, respectively. These data sets were created by going through the original set and selecting every thousandth, hundredth, tenth, fifth, and every second and third sentence, respectively.

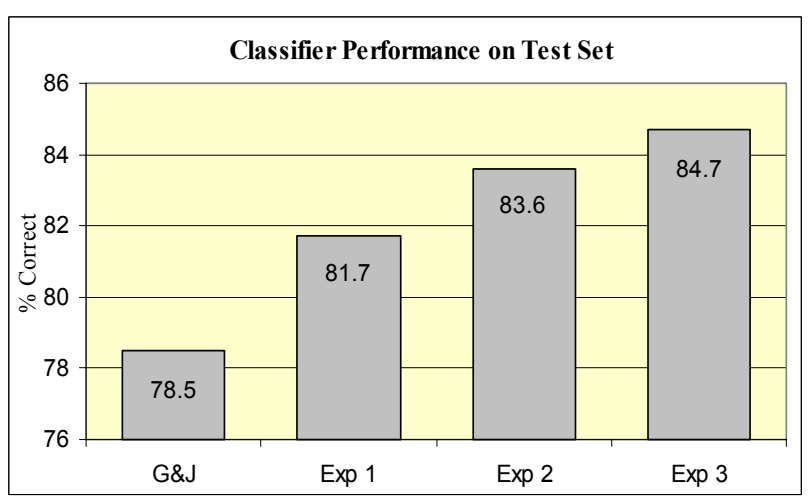

Figure 2. Performance of models on test data using hand annotated frame element boundaries. G\&J refers to the results of Gildea and Jurafsky (2002). Exp 1 incorporates feature sets 0-7 from Table 1; Exp 2 feature sets 0-9; Exp 3 features 0-11.

\subsection{Results}

Figure 2 shows the results of our experiments alongside those of (Gildea and Jurafsky, 2002) on identical held out test sets. The difference in performance between each classifier is statistically significant at $(\mathrm{p}<0.01)$ (Mitchell, 1997), with the 
exception of Exp 2 and Exp 3, whose difference is statistically significant at $(\mathrm{p}<0.05)$.

Table 2. Effect of different smoothing parameter (std. dev.) values on classification performance.

\begin{tabular}{|l|l|}
\hline Std. Dev. & \% Correct \\
\hline 1 & 79.9 \\
\hline 2 & 82.1 \\
\hline 4 & 81.9 \\
\hline
\end{tabular}

Table 2 shows the effect of varying the standard deviation of the Gaussian priors used for smoothing in Experiment 1. The difference in performance between the classifiers trained using standard deviation 1 and 2 is statistically significant at $(\mathrm{p}<0.01)$.

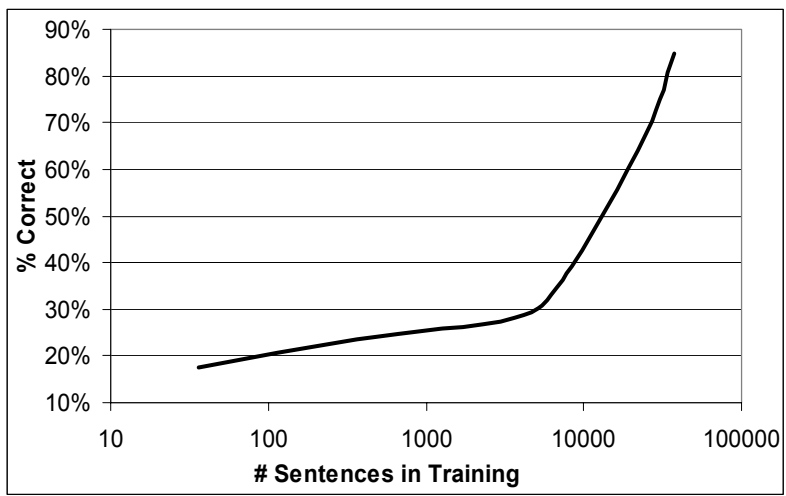

Figure 3. Effect of training set size on semantic role classification.

Figure 3 shows the change in performance as a function of training set size. Classifiers were trained using the full set of features described for Experiment 3.

Table 3 shows the confusion matrix for a subset of semantic roles. Five roles were chosen for presentation based upon their high contribution to classifier error. Confusion between these five account for $27 \%$ of all errors made amongst the 120 possible roles. The tenth role, other, represents the sum of the remaining 115 roles. Table 4 presents example errors for five of the most confused roles.

\subsection{Discussion}

It is clear that the ME models improve performance on frame element classification. There are a number of reasons for this improvement.

First, for this task the log-linear model employed in the ME framework is better than the linear interpolation model used by Gildea and Jurafsky. One possible reason for this is that semantic role classification benefits from the ME model's bias for more uniform probability distributions that satisfy the constraints placed on the model by the training data.

Another reason for improved performance comes from ME's simpler design. Instead of having to worry about finding proper backoff strategies amongst distributions of features subsets, ME allows one to include many features in a single model and automatically adjusts the weights of these features appropriately.

Table 3. Confusion matrix for five roles which contribute most to overall system error. Columns refer to actual role. Rows refer to the model's hypothesis. Other refers to combination of all other roles.

\begin{tabular}{|c|c|c|c|c|c|c|c|}
\hline & Area & Spkr & Goal & Msg & Path & Other & Prec. \\
\hline Area & 98 & & 6 & & 18 & 16 & 0.710 \\
\hline Spkr & & 373 & & 23 & & 41 & 0.853 \\
\hline Goal & 11 & & 431 & & 28 & 50 & 0.828 \\
\hline Msg & & 18 & 1 & 315 & & 33 & 0.858 \\
\hline Path & 32 & & 36 & & 415 & 41 & 0.791 \\
\hline Other & 15 & 21 & 26 & 24 & 33 & 5784 & 0.979 \\
\hline Recall & 0.628 & 0.905 & 0.862 & 0.87 & 0.84 & 0.969 & \\
\hline
\end{tabular}

Also, because the ME models find weights for many thousands of features, they have many more degrees of freedom than the linear interpolated models of Gildea and Jurafsky. Although many degrees of freedom can lead to overfitting of the training data, the smoothing procedure employed in our experiments helps to counteract this problem. As evidenced in Table 2, by optimizing the standard deviation used in smoothing the ME models are able to show significant increases in performance on held out test data.

Finally, by including in our model sentencelevel pattern features and information about previous classes, global information can be exploited for improved classification. The accuracy gained by including such global information confirms the intuition that the semantic role of an element is much related to the entire sentence of which it is a part.

Having discussed the advantages of the models presented here, it is interesting to look at the errors that the system makes. It is clear from the confusion matrix in Table 3 that a great deal of the system error comes from relatively few semantic 
roles. ${ }^{4}$ Table 4 offers some insight into why these errors occur. For example, the confusions exemplified in 1 and 2 are both due to the fact that the particular phrases employed can be used in multiple roles (including the roles hypothesized by the system). Thus, while "across the counter" may be considered a goal when one is talking about a person and their head, the same phrase would be considered a path if one were talking about a mouse who is running.

Table 4. Example errors for five of the most often confused semantic roles

\begin{tabular}{|l|l|l|l|}
\hline & Actual & Proposed & Example Sentence \\
\hline 1 & Goal & Path & $\begin{array}{l}\text { The barman craned his head } \\
\text { across the counter. }\end{array}$ \\
\hline 2 & Area & Path & $\begin{array}{l}\text { Mr. Glass began hallucinating, } \\
\text { throwing books around the } \\
\text { classroom. }\end{array}$ \\
\hline 3 & Message & Speaker & $\begin{array}{l}\text { Debate lasted until 20 Septem- } \\
\text { ber, opposition being voiced } \\
\text { by a number of Italian and } \\
\text { Spanish prelates. }\end{array}$ \\
\hline 4 & Addressee & Speaker & $\begin{array}{l}\text { Furious staff claim they were } \\
\text { even called in from holiday to } \\
\text { be grilled by a specialist secu- } \\
\text { rity firm }\end{array}$ \\
\hline 5 & Reason & Evaluee & $\begin{array}{l}\text { We cannot but admire the } \\
\text { efficiency with which she } \\
\text { took control of her own life. }\end{array}$ \\
\hline
\end{tabular}

Examples 3 and 4, while showing phrases with similar confusions, stand out as being errors caused by an inability to deal with passive sentences. Such errors are not unexpected; for, even though the voice of the sentence is an explicit feature, the system suffers from the paucity of passive sentences in the data (approximately 5\%).

Finally, example 5 shows an error that is based on the difficult nature of the decision itself (i.e., it is unclear whether "the efficiency" is the reason for admiration, or what is being admired). Often times, phrases are assigned semantic roles that are not obvious even to human evaluators. In such cases it is difficult to determine what information might be useful for the system.

Having looked at the types of errors that are common for the system, it becomes interesting to examine what strategy may be best to overcome such errors. Aside from new features, one solution is obvious: more data. The curve in Figure 2 shows that there is still a great deal of performance to be gained by training the current ME models on

\footnotetext{
${ }^{4} 44 \%$ of all error is due to confusion between only nine roles.
}

more data. The slope of the curve indicates that we are far from a plateau, and that even constant increases in the amount of available training data may push classifier performance above $90 \%$ accuracy.

Having demonstrated the effectiveness of the ME approach on frame element classification given hand annotated frame element boundaries, we next examine the value of the approach given automatically identified boundaries.

\section{Frame Element Identification}

Gildea and Jurafsky equate the task of locating frame element boundaries to one of identifying frame elements amongst the parse tree constituents of a given sentence. Because not all frame element boundaries exactly match constituent boundaries, this approach can perform no better than $86.9 \%$ (i.e. the number of elements that match constituents (6864) divided by the total number of elements (7899)) on the test set.

\subsection{Features}

Frame element identification is a binary classification problem in which each constituent in a parse tree is described by a feature vector and, based on that vector, tagged as either a frame element or not. In generating feature vectors we use a subset of the features described for role tagging as well as an additional path feature.

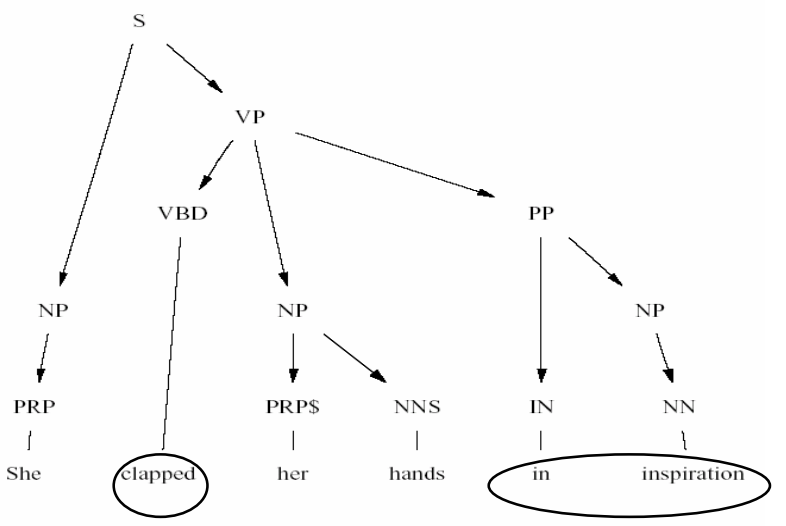

Figure 4. Generation of path features used in frame element tagging. The path from the constituent "in inspiration" to the target predicate "clapped" is represented as the string PP $\uparrow \mathrm{VP} \downarrow$ VBD.

Gildea and Jurafsky introduce the path feature in order to capture the structural relationship between a constituent and the target predicate. The 
Table 5. Results of frame element identification. G\&J represents results reported in (Gildea and Jurafsky, 2002), ME results for the experiments reported here. The second column shows precision, recall, and F-scores for the task of frame element identification, the third column for the combined task of identification and classification.

\begin{tabular}{|l|l|l|l|l|l|l|}
\hline \multirow{2}{*}{ Method } & \multicolumn{3}{c|}{ FE ID only } & \multicolumn{3}{c|}{ FE ID + FE Classification } \\
\cline { 2 - 7 } & Precision & Recall & F-Score & Precision & Recall & F-Score \\
\hline G\&J Boundary id + baseline role labeler & .726 & .631 & .675 & .67 & .468 & .551 \\
\hline ME Boundary id + ME role labeler & .736 & .679 & .706 & .6 & .554 & .576 \\
\hline
\end{tabular}

path of a constituent is represented by the nodes through which one passes while traveling up the tree from the constituent and then down through the governing category to the target. Figure 4 shows an example of this feature for a frame element from the sentence presented in Figure 1.

\subsection{Experiments}

We use the ME formulation described in Section 3.2 to build a binary classifier. The classifier features follow closely those used in Gildea and Jurafsky. We model the data using the feature sets: $f(f e$, path), f(fe, path, tar), and $f(f e$, head, tar), where $f e$ represents the binary classification of the constituent. While this experiment only uses three feature sets, the heterogeneity of the path feature is so great that the classifier itself uses $1,119,331$ unique binary features.

With the constituents having been labeled, we apply the ME frame element classifier described above. Results are presented using the classifier of Experiment 1, described in section 3.3. We then investigate the effect of varying the number of constituents used for training on identification performance. Five data sets of approximately 100,000 $10,000,1,000$, and 100 constituents were generated from the original set by random selection and used to train ME models as described above.

\subsection{Results}

Table 5 compares the results of Gildea and Jurafsky (2002) and the ME frame element identifier on both the task of frame element identification alone, and the combined task of frame element identification and classification. In order to be counted correct on the combined task, the constituent must have been correctly identified as a frame element, and then must have been correctly classified into one of the 120 semantic categories.

Recall is calculated based on the total number of frame elements in the test set, not on the total number of elements that have matching parse constituents. Thus, the upper limit is $86.9 \%$, not
$100 \%$. Precision is calculated as the number of correct positive classifications divided by the number of total positive classifications.

The difference in the F-scores on the identification task alone and on the combined task are statistically significant at the $(\mathrm{p}<0.01)$ level ${ }^{5}$. The accuracy of the ME semantic classifier on the automatically identified frame elements is $81.5 \%$, not a statistically significant difference from its performance on hand labeled elements, but a statistically significant difference from the classifier of Gildea and Jurafsky $(2002)(\mathrm{p}<0.01)$.

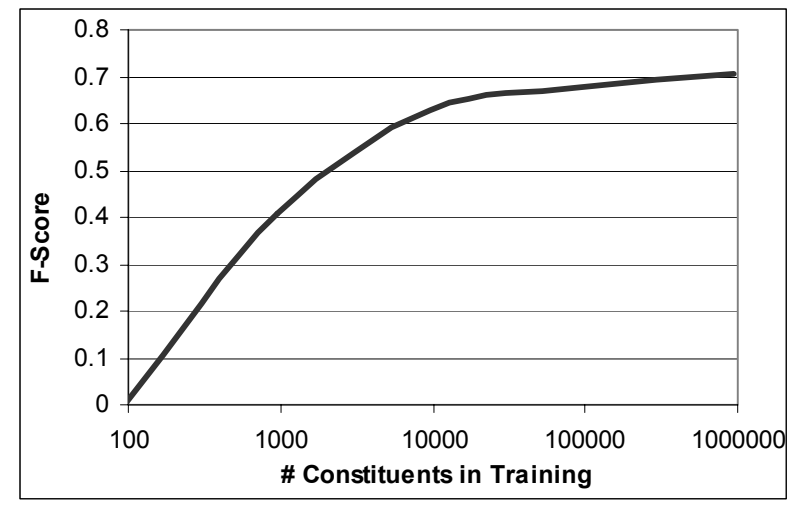

Figure 5. Effect of training set size on frame element boundary identification.

Figure 5 shows the results of varying the training set size on identification performance. For each data set, thresholds were chosen to maximize F-Score.

\subsection{Discussion}

It is clear from the results above that the performance of the ME model for frame element classification is robust to the use of automatically identified frame element boundaries. Further, the ME

\footnotetext{
${ }^{5}$ G\&J's results for the combined task were generated with a threshold applied to the FE classifier (Dan Gildea, personal communication). This is why their precision/recall scores are dissimilar to their accuracy scores, as reported in section 3 . Because the ME classifier does not employ a threshold, comparisons must be based on F-score.
} 
framework yields better results on the frame element identification task than the simple linear interpolation model of Gildea and Jurafsky. This result is not surprising given the discussion in Section 3.

What is striking, however, is the drastic overall reduction in performance on the combined identification and classification task. The bottleneck here is the identification of frame element boundaries. Unlike with classification though, Figure 5 indicates that a plateau in the learning curve has been reached, and thus, more data will not yield as dramatic an improvement for the given feature set and model.

\section{Conclusion}

The results reported here show that ME models provide higher performance on frame element classification tasks, given both human and automatically identified frame element boundaries, than the linear interpolation models examined in previous work. We attribute this increase to the benefits of the ME framework itself, the incorporation of sentence-level syntactic patterns into our feature set, and the use of previous tag information to find the most probable sequence of roles for a sentence.

But perhaps most striking in our results are the effects of varying training set size on the performance of the classification and identification models. While for classification, the learning curve appears to be still increasing with training set size, the learning curve for identification appears to have already begun to plateau. This suggests that while classification will continue to improve as the FrameNet database gets larger, increased performance on identification will rely on the development of more sophisticated models.

In future work, we intend to apply the lessons learned here to the problem of frame element identification. Gildea and Jurafsky have shown that improvements in identification can be had by more closely integrating the task with classification (they report an F-Score of .719 using an integrated model). We are currently exploring a ME approach which integrates these two tasks under a tagging framework. Initial results show that significant improvements can be had using techniques similar to those described above.

\section{Acknowledgments}

The authors would like to thank Dan Gildea who generously allowed us access to his data files and Oliver Bender for making the MEtagger software available. Finally, we thank Franz Och whose help and expertise was invaluable.

\section{References}

O. Bender, K. Macherey, F. J. Och, and H. Ney. 2003. Comparison of Alignment Templates and Maximum Entropy Models for Natural Language Processing. Proc. of EACL-2003. Budapest, Hungary.

A. Berger, S. Della Pietra and V. Della Pietra, 1996. A Maximum Entropy Approach to Natural Language Processing. Computational Linguistics, vol. 22, no. 1.

S. F. Chen and R. Rosenfeld. 1999. A Gaussian prior for smoothing maximum entropy models. Technical Report CMUCS -99-108, Carnegie Mellon University

M. Collins. 1997. Three generative, lexicalized models for statistical parsing. Proc. of the $35^{\text {th }}$ Annual Meeting of the ACL. pages 16-23, Madrid, Spain.

J. N. Darroch and D. Ratcliff. 1972. Generalized iterative scaling for log-linear models. Annals of Mathematical Statistics, 43:1470-1480.

C. Fillmore 1976. Frame semantics and the nature of language. Annals of the New York Academy of Sciences: Conference on the Origin and Development of Language and Speech, Volume 280 (pp. 20-32).

D. Gildea and D. Jurafsky. 2002. Automatic Labeling of Semantic Roles, Computational Linguistics, 28(3) 245-288 14.

T. Mitchell. 1997. Machine Learning. McGrawHill International Editions, New York, NY. Pages 143-145.

F.J. Och. 2002. Yet another maxent toolkit: YASMET. www-i6.informatik.rwthaachen.de/Colleagues/och/. 